\title{
LOS INMIGRANTES EXTRANJEROS EN TERUEL ${ }^{1}$
}

\author{
Pascual Rubio Terrado \\ Facultad de Ciencias Sociales y Humanas, Universidad de Zaragoza \\ pasrubio@unizar.es
}

\begin{abstract}
Resumen: La llegada de inmigrantes extranjeros a la provincia de Teruel es más tardía que en el conjunto de España, pero hoy, casi todos sus municipios incorporan población extranjera en el padrón de habitantes. La inmigración se ha convertido en el proceso demográfico con mayor impacto territorial, de la misma manera que la emigración lo fue hasta hace menos de un decenio. Conocer las características de ese grupo humano y su distribución territorial, evaluar los efectos demográficos y territoriales de su llegada y valorar la percepción que sobre el proceso inmigratorio tiene la población turolense, son los objetivos básicos de este trabajo. Se parte de la premisa de que cuando el deterioro demográfico de un territorio rebasa la capacidad de autoregeneración del grupo humano, esto se convierte en una restricción muy robusta para el desarrollo, por ello, su revitalización humana y funcional precisa mecanismos que activen un saldo migratorio positivo sostenido.
\end{abstract}

Palabras clave: Inmigración, extranjeros, Teruel, consecuencias demográficas y territoriales.

\begin{abstract}
The arrival of foreign immigrants to the province of Teruel is later than in the whole of Spain, but today, almost all municipalities incorporate foreign population in the population census. The immigration has become the demographic process with the greatest regional impact, in the same way that the emigration was until less than a decade. To know the characteristics of that group of people and their territorial distribution, to evaluate the demographic and territorial arrival and to assess the perception of the immigration process is population Teruel, are the basic objectives of this paper. We start from the premise that

Recibido: 2-11-10. Aceptado: 2-2-12.

${ }^{1}$ En este artículo presentamos los principales resultados del proyecto "Población rural y revitalización de los territorios rurales frágiles. El caso del sistema territorial turolense", financiado por la Fundación Universitaria Antonio Gargallo (convocatoria 2007) y la Comarca Comunidad de Teruel.
\end{abstract}


when the demographic decline of an area exceeds the capacity of selfregeneration of the human group, this becomes a very strong constraint to development, therefore, its functional and human revitalization needs mechanisms that activate a sustained migratory positive balance.

Keyworks: Immigration, foreigners, Teruel, demographic and territorial implications.

\section{Introducción}

En las etapas con dinamismo escaso de la actividad económica, la población tiende a crecer poco y si lo hace se incrementa la presión sobre los recursos. La válvula que se activa entonces es la emigración, lo que mejora en el corto plazo el capital territorial per capita disponible, si bien, en el largo, de mantenerse la tendencia, puede causar deterioro de la estructura demográfica, despoblamiento y desorganización productiva y de la red asentamientos. Además, en línea con lo señalado por Sáez (2008), la falta de población se convierte en una restricción muy robusta para el desarrollo; Domínguez (2003) indica que la economía política enseña que no puede haber desarrollo sin incremento de población. Por eso, cuando el deterioro rebasa la capacidad de autoregeneración del grupo, la revitalización humana precisa mecanismos que activen un saldo migratorio positivo sostenido. El problema, en todo caso, deriva de que la revitalización se produzca sin creación de nueva riqueza, lo que puede conducir a que aquella, además de coyuntural, sea reversible.

La inmigración y sus repercusiones sociales, culturales, económicas y territoriales es un tema bien tratado desde campos como la sociología, el derecho, la educación, la economía y la geografía, pues, como indica Cohen (2009), hay pocos objetos de estudio en las ciencias sociales que hayan concitado una convergencia de intereses tan importante. Sin ánimo de exhaustividad, destacamos los trabajos de AmuedoDorantes y De la Rica (2005), Carrasco y Ortega (2005), García Sanz (2006), Serrano (2006), González (2008), Roquer y Blay (2008), Colectivo IOE (2008, 2010a y 2010b), Cánoves y Blanco (2009), Casado (2009), Cebrián et alii (2010), Bayona y Gil (2010) y Rodríguez (2010), entre otros. Todos enfatizan la existencia, desde finales de la última década del siglo pasado, de un intenso proceso de llegada de inmigrantes extranjeros que está causando un fuerte impacto en el sistema territorial español. En el caso particular del territorio rural, el proceso explica por qué en una porción muy significativa del mismo se ha detenido el deterioro demográfico e iniciado una tendencia hacia la estabilidad y/o crecimiento. Este proceso, aunque con ritmo e intensidad distintos, también se observa en Teruel, pero ha sido poco estudiado, salvo la 
participación del colectivo latinoamericano en la mejora del capital social provincial (Martín-Hernández et alii, 2007).

Desde la idea de que la inmigración, parafraseando a Bel y Gómez (2001), más que un reto es una oportunidad para Teruel, nuestros objetivos se orientan, en primer lugar, a analizar cómo es, a qué aspiran y cómo perciben el territorio turolense los residentes extranjeros, en segundo, a conocer cómo valoran los turolenses la inmigración y, finalmente, a evaluar las consecuencias de la inmigración; los dos primeros han precisado de un intenso trabajo de campo, materializado en la realización de entrevistas a inmigrantes extranjeros y encuestas telefónicas a empadronados de nacionalidad española; la consecución del tercero se ha basaso en la revisión de la información de Censos, Padrones de Población y Estadística de Variaciones Residenciales. Combinamos, pues, un enfoque general-cualitativo (o social, teniendo en cuenta que los inmigrantes son el sujeto), con otro estructural-cuantitativo (o socioeconómico y territorial, tomando a los inmigrantes como objeto).

\section{La evolución demográfica turolense}

Las ligazones entre población y actividad económica, resueltas en un flujo de doble dirección que acarrea que los cambios en el estado de una variable afecten a la funcionalidad y comportamiento de la otra, son el punto de partida de nuestra reflexión; añadido a eso, entendemos que ambas mantienen relaciones con otras que generan circuitos de retroalimentación capaces de exportar los comportamientos demo-económicos a otros componentes del sistema territorial, sobre el que pueden promover modificaciones en su actividad, organización y estado. Este planteamiento explica el porqué del deterioro territorial turolense durante la larga etapa pasada de vaciado demográfico y, sobre todo, las consecuencias del reciente cambio de tendencia.
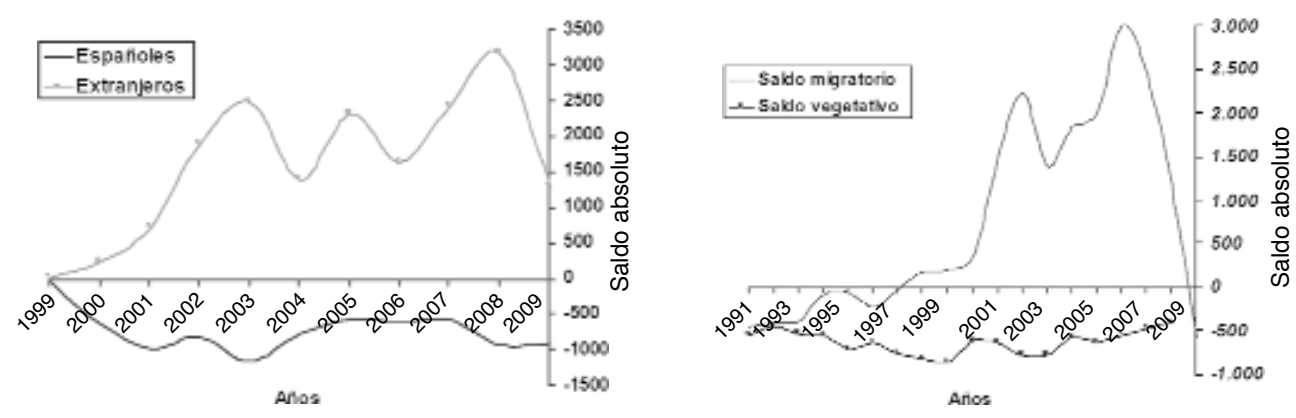

Figura 1. Variaciones residenciales (A) y evolución de los saldos migratorio y vegetativo (B). Elaboración propia. 
Durante los últimos doscientos años, la acción sobre el espacio ha respondido a un modelo de carácter naturalizado y el desarrollo y localización de las actividades económicas ha tendido a depender, como señala Sáez (2007), de las ventajas de las materias de primera naturaleza. Por eso, mientras el entorno ha sido más dinámico, en Teruel se ha producido una pérdida de competitividad que ha causado emigración. Aunque hasta principios del siglo XX existió un cierto crecimiento demográfico (vid figura 3A), desde la década de los veinte, cuando se hizo más patente su carácter de isla rodeada de territorios beneficiados por la emergencia de los nuevos factores de localización industrial, básicamente el sistema de transportes, la provincia empezó a perder población, tendencia que se ha mantenido hasta hace menos de un decenio. La debacle demográfica turolense, con la excepción de unos pocos núcleos urbanos y semiurbanos, ha ganado en intensidad con el paso del tiempo; a ello se ha sumado la concentración paulatina de la población en los núcleos más grandes.

Pero desde finales de los noventa del siglo XX, Teruel se ha convertido en receptor neto de recursos humanos (vid figura 1A), dada una entrada regular de inmigrantes extranjeros que ha compensado la emigración de españoles y el saldo natural estructuralmente negativo que sigue presentando la población (vid figura 1B). La inmigración ha pasado a ser un proceso demográfico importante, con alto impacto sobre la evolución demográfica, como lo demuestra la fuerte correlación entre los saldos migratorio y demográfico durante el periodo 2001 a 2008 (vid figura 3B), y sobre la población activa; por el contrario, su incidencia sobre el saldo vegetativo no está siendo inmediata.

\section{Los inmigrantes extranjeros en Teruel}

La llegada de inmigrantes extranjeros es un proceso reciente y poco conocido, no tanto en lo relacionado con los valores cuantitativos del mismo (sobre los que informan Censos y Padrones de Población y la Estadística de Variaciones Residenciales), cuanto en lo referido a otros aspectos cualitativos (motivos y ruta de la inmigración, grado de participación en las actividades socio-culturales y de integración en la vida local, problemas de integración, condiciones de calidad de vida, grado de satisfacción con la estancia, previsión de cambios futuros en la residencia,...). De la misma manera, tampoco hay fuentes para conocer sobre la percepción que este proceso suscita entre la población turolense (valoración sobre la llegada de inmigrantes y relaciones que se mantienen con ellos, integración de este colectivo, aspectos positivos y negativos de la inmigración, preocupación por la llegada de más inmigrantes,...).

Para superar esas limitaciones, hemos realizado entrevistas a inmigrantes y encuestas a población española. Se han cumplimentado un total de 117 entrevistas a extranjeros de entre 18 y 65 años (el 0,84\% del colectivo a 1 de enero de 2008) durante el 
primer semestre de ese año, en 47 municipios. Para la elección de la muestra se han tenido en cuenta los residentes extranjeros en Teruel y su distribución a partir de los datos proporcionados por el Padrón Municipal de Habitantes a 1 de enero de 2007; los municipios de realización se han seleccionado aleatoriamente, partiendo de tres restricciones iniciales, que todos los tamaños de municipio y categorías de ruralidad estén representados de forma proporcional a la presencia de inmigrantes y que las diez capitales comarcales figuren en la muestra. Las entrevistas se han realizado en asociaciones de atención a inmigrantes (como ATIS), en locales de organizaciones sindicales, en centros sociales y en la calle. ${ }^{2}$

También hemos efectuado 585 encuestas telefónicas, dirigidas a empadronados de nacionalidad española (un 0,52\% de los mayores de edad), elegidas al azar en 125 municipios y distribuidas según los mismos criterios anteriores.

\subsection{Características básicas del colectivo inmigrante}

Por origen, existe un predominio de inmigrantes europeos, especialmente rumanos; por edad, mayoritariamente se trata de individuos de entre 31-45 años, de origen urbano ( $88 \%$ en general y 77,8 entre los africanos) y con nivel de estudios medio-alto (más del $50 \%$ dicen tener estudios secundarios concluidos y un $18 \%$ universitarios). Viven prioritariamente formando unidades familiares de padres-hijos (un $70 \%$ están casados o viven en pareja) o compartiendo vivienda con otros compatriotas (categoría más frecuente entre los africanos). Desde el punto de vista de la antigüedad de la residencia, un 60,5 \% dice hacer tres o más años que están en Teruel y un 12,3 uno o menos.

En lo referido al periplo migratorio y a las razones de por qué emigraron, motivos de tipo económico (oportunidades laborales) y personales (presencia de familiares y/o amigos que han facilitado la existencia de redes informales de acogida inicial) son los argumentos más frecuentes (86,6 \% conjunto de las respuestas). La mayoría $(53,8 \%)$ han llegado directamente a la provincia de Teruel y sólo un $14,5 \%$ han estado anteriormente en otros lugares $(27,7 \%$ entre los africanos).

Según sectores de actividad en los países de origen, comercio, industria y otros servicios son los más significativos; destaca el bajo porcentaje de los que señalan la construcción y la agricultura, con la excepción de los africanos (57,7\%). Atendiendo a la cualificación profesional, un $37 \%$ manifiesta no poseer ninguna en espacial $(85,7 \%$ entre los africanos) y sólo el 20,6 \% la valora de media a alta (33,3 entre los europeos).

${ }^{2}$ Agradecemos a los responsables de esas organizaciones la ayuda brindada, muy en especial a Ángeles Villarroya (UGT-Teruel). Así mismo, por la profesionalidad de su trabajo, damos las gracias a los encuestadores de la empresa GEOTER. 
Sobre la actividad y las condiciones de trabajo en Teruel, el $38 \%$ confiesa trabajar en la construcción y un $20 \%$ en la hostelería, con contratos temporales (52\%), mayoritariamente a jornada completa (82\%) y con condiciones que se han mantenido como mínimo durante el último año en un $55 \%$ de los casos $(76,2 \%$ entre los europeos). El número medio de puestos de trabajo desempeñados desde su llegada es de dos, por lo que, dado lo reciente del proceso, pensamos que la movilidad laboral es más alta que entre los nacionales (entre los latinoamericanos destaca la secuencia hostelería/servicio doméstico, entre los europeos industria/hostelería y entre los africanos sector agrario/construcción). Si bien no perciben posibilidades claras de promocionar, la mayoría se encuentran satisfechos en su puesto de trabajo, tanto por horarios, como por salario, condiciones laborales y relaciones con los compañeros; en un ranking de 1 (pésimas-muy malas) a 10 (excelentes-muy buenas), la mayoría de las respuestas oscilan entre 7 y 9 (aunque los latinoamericanos son los más críticos -media de 6,5-). Pero un 39,3\% aspira a cambiar de trabajo $(58,1 \%$ entre los latinoamericanos, aunque pocos determinan hacia qué sector o actividad), destacando la proporción de quienes desean establecerse como autónomos en el mismo sector en el que desarrollan actualmente su trabajo y sin cambiar de lugar de residencia $(60,7$ $\%$, aunque para los africanos es de 33,3), lo que indica una inclinación a asentarse en este territorio; entre quienes sí lo revelan, las razones de tipo laboral y/o personal son los argumentos más destacados. Pocos han recibo formación laboral más allá de la experiencia que proporciona el desempeño del puesto de trabajo.

En lo relacionado con la integración y condiciones generales de vida, manifiestan participar poco en las actividades sociales de los lugares donde viven (entre los de origen latinoamericano y europeo las respuestas afirmativas son más frecuentes y también entre los residentes en municipios donde viven pocos inmigrantes y/o no se han consolidado redes por nacionalidad), pero sí dicen sentirse integrados, lo que se justifica porque no perciben problemas de rechazo entre la población local, con la que la relación es muy buena para un $64 \%$; entre los de origen africano, sólo el idioma ha sido un problema importante, si bien, aprecian un apoyo social creciente al proceso inmigratorio en Teruel. La puntuación otorgada a los equipamientos sociales $(8,0$ sobre 10$)$, educativos $(7,8)$ y sanitarios $(9,0)$, la calidad de la vivienda ocupada $(7,6)$, oportunidades laborales $(6,5)$, transportes $(6,8)$, oferta comercial $(7,2)$ y seguridad ciudadana $(9,7)$ son los factores que justifican una opinión de que su calidad de vida es elevada. La mayor parte (87,2 \%) ocupan una vivienda en alquiler, aunque un 23,4\% mantiene expectativas de adquirirla en propiedad en un futuro próximo.

Desde el punto de vista de la actitud hacia el territorio, el 96,6\% dicen encontrarse a gusto; de nuevo, la tranquilidad y seguridad, las posibilidades de trabajo, ser un lugar agradable y las comodidades de la vivienda justifican esa apreciación. Pocos son los que declaran plantearse el retorno a su país (un 7,8 \% y sólo tras la jubila- 
ción). La valoración del futuro en lo relativo a las condiciones de integración y de apoyo social e institucional es positiva para casi el 50 \%; sólo los escenarios laboral y económico futuros parece indicar cierta incertidumbre, lo que relacionamos con el momento de realización de las entrevistas, cuando la actual situación de crisis ya era evidente, las tasas de ocupación indicaban un repliegue y las de paro un repunte, sobre todo en la construcción.

En conclusión, destacamos que se trata de un colectivo compuesto por individuos jóvenes y volcados hacia el mercado laboral, se siente bien tratado e integrado en las sociedades locales (aunque la apreciación disminuye cuando residen en los medios urbano y rururbano), valora positivamente la mejora que para su calidad de vida ha supuesto la emigración, aspira a asentarse en este territorio (porque percibe suficientes oportunidades laborales y una actitud favorable por parte de la población local), y muestra un grado alto de fidelidad al mismo, como lo demuestra el elevado porcentaje de los que están residiendo de forma ininterrumpida desde hace, como mínimo, tres años (eso sí, por colectivos, lo es mucho más entre los europeos y latinoamericanos que entre los africanos, probablemente porque los dos primeros grupos tienden a vivir más formando núcleos familiares y su estabilidad laboral también es mayor).

\subsection{Valoración del proceso inmigratorio}

El proceso es valorado de forma positiva o muy positiva por el 58,3\% de los encuestados y sólo un 5,3\% lo consideran muy negativo. La mejoría de la actividad económica (un 18,5\% de las respuestas), la dinamización del mercado de trabajo $(19,0 \%)$ y, en especial, el impulso demográfico que otorga $(32,3 \%)$, son los aspectos mejor valorados; paralelamente, un $8,9 \%$ señalan que no hay ninguno y otro 40,5 $\%$ manifiesta cierta inquietud por la intensidad del ritmo actual, de lo que deriva una cierta corriente de opinión de que la capacidad local de absorción de inmigrantes extranjeros, sobre todo en los pueblos más pequeños, tiene un límite; pero también se considera que el techo máximo no se ha alzado. Entre los negativos, destacan competencia por el empleo $(30,3 \%)$, problemas de convivencia $(37,1 \%)$ y aumento de la delincuencia e inseguridad ciudadana ( $46,7 \%)$; en general, esas opiniones mantienen un alto grado de coincidencia con los mensajes más genéricos que sobre el proceso transmiten los medios de comunicación.

En lo relativo a la convivencia, sólo el $22,6 \%$ señala tener un trato fluido con los inmigrantes, sobre todo en el trabajo (los hombres) y en la vida cotidiana (las mujeres) y muy poco en actividades culturales y de ocio. Aunque no parecen detectar un déficit en la integración, la dificultad para evaluar un concepto que reconocemos es impreciso, explica que las respuestas estén muy repartidas, ya que si bien para el 
$36,9 \%$ es suficiente y excelente para un 23,4, otro 31,6 piensa que es insatisfactoria. Con todo, creemos que la proximidad cultural de cada grupo a la población española tiene mucho que ver en la percepción sobre la integración (aparece como factor explicativo en el 20,3\% de las respuestas; si bien, la actitud general no conflictiva es el aspecto más destacado (36,0\%).

Por tamaño de municipio, en los de menos de 1.000 habitantes se observan las valoraciones más positivas (66,6\%); ello deriva de la visualización más explícita de su carácter de rejuvenecedor demográfico (46,9\%), de los menores índices de preocupación porque sigan llegando inmigrantes (34,6\%) y de relaciones más fluidas en el ámbito personal. El porcentaje se reduce cuando aumenta el tamaño; en los de más de 5.000, sólo el 50,2 \% hace una valoración positiva y el 24,4 cita el factor de rejuvenecedor, mientras que la preocupación porque sigan llegando inmigrantes la menciona el $62,1 \%$ (destacando con un $57,2 \%$ la delincuencia y la competencia por el empleo); curiosamente, la valoración del grado de integración crece, quizás porque la presión que ejercen sobre la cultura local se reduce y lo mismo su visibilidad relativa, al difuminarse entre un grupo humano más numeroso.

Por nacionalidades, los latinoamericanos son los mejor valorados (algo más de 5,5 puntos sobre 10), seguidos de los europeos, mientras que a los africanos corresponde la peor posición (3,6 puntos). La mayor o menos proximidad cultural, nuevamente, influye en la respuesta.

\subsection{Consecuencias demográficas y territoriales}

El colectivo de inmigrantes extranjeros estaba formado por 498 personas en 1996 (0,4 \% de la población total); en 2001, las cifras se habían multiplicado por tres; posteriormente el ritmo ha ganado intensidad (18.369 personas en 2009, el 12,5\%). Los factores que explican esta evolución coinciden con los observados en el resto del país; en lo básico, una demanda laboral insuficientemente satisfecha por el mercado local, principalmente en obra pública, construcción, restauración, hostelería, agricultura, servicio doméstico y atención de la población dependiente (concentran casi el $90 \%$ del empleo total para este colectivo en Teruel); a lo anterior se suma el efecto llamada ligado al alto nivel de crecimiento del PIB en los últimos 15 años, que ha arrastrado tras de sí al mercado de trabajo. Pero no se debe olvidar que la propia inmigración ha retroalimentado sobre el crecimiento de esa magnitud; según un informe de la Oficina Económica de Presidencia del Gobierno, el 30\% del aumento del PIB durante la última década debe ser asignado a la inmigración y el porcentaje se eleva hasta el 50\% de limitar el análisis a los últimos cinco años. 


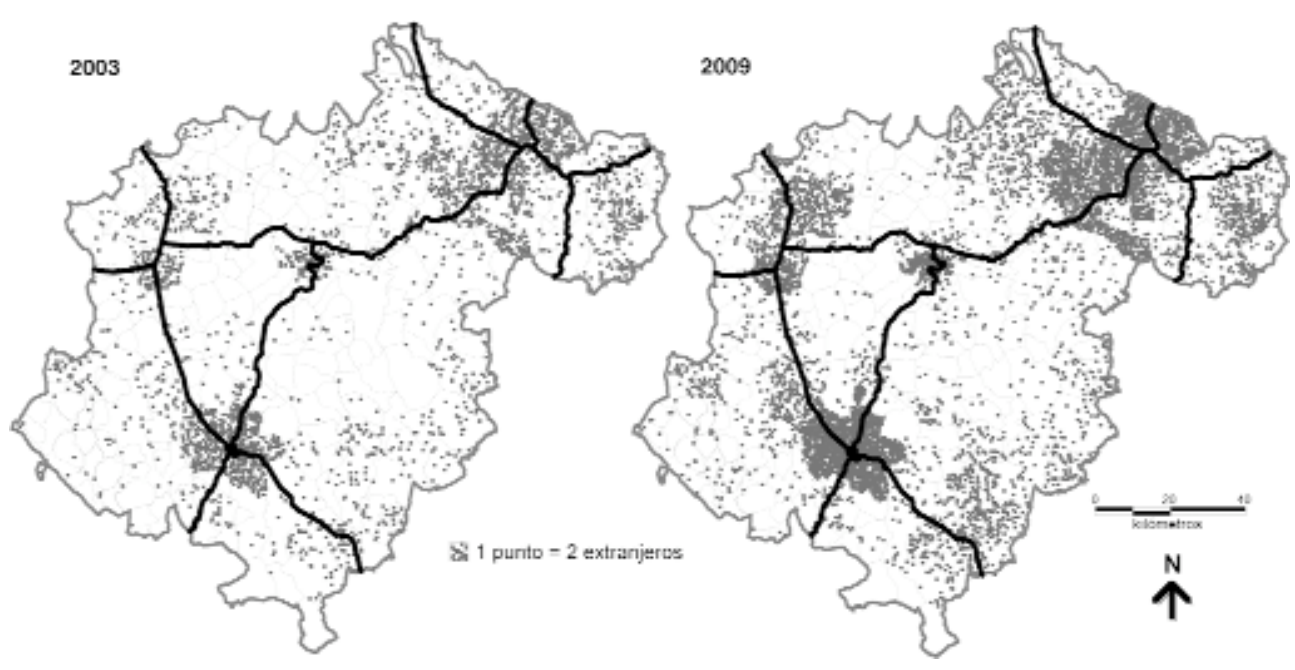

Figura 2. Distribución de la población extranjera. Elaboración propia.

Aunque las tendencias generales son similares en todas las comarcas, las discontinuidades temporales en el ritmo de llegada y espaciales en el reparto, han originado ciertos desequilibrios (vid figura 2). Andorra-S ${ }^{\underline{a}}$ de Arcos, Matarraña, Bajo Aragón y Comunidad de Teruel han sido las últimas en incorporarse al proceso. GúdarJavalambre (16,8 \%), Jiloca $(13,9)$ y Bajo Aragón $(13,7)$ tienen el mayor porcentaje de participación de la población extranjera sobre la total; en la primera, el bajo nivel demográfico de partida y el dinamismo de los sectores turístico y de la construcción justifican esta circunstancia, en las otras dos interviene el carácter industrial dinámico de sus cabeceras y subcabeceras. En el otro extremo figuran $S^{\underline{a}}$ de Albarracín (9,2 \%), Cuencas Mineras $(9,6)$ y Bajo Martín $(8,4)$; la primera, es la más deprimida, envejecida y en la que la transición desde un modelo productivo centrado en el sector primario hacia otro postagrario se encuentra menos avanzada, por lo tanto, el dinamismo del mercado de trabajo es escaso; la crisis de la minería, con la consecuente destrucción de tejido productivo, explica la menor presencia de extranjeros en las otras dos, circunstancia que coincide con ser en las que antes se ha agotado el proceso (desde 2005 presentan índices negativos de variación en la población extranjera residente).

De la presencia de este colectivo derivan interesantes consecuencias demográficas y territoriales: ha contribuido a reforzar algunas tendencias ya percibidas a finales de los noventa, como resistencia a la desaparición de pequeños núcleos de población (en los que unos pocos extranjeros aseguran su pervivencia), concentración progresiva de la población y del crecimiento demográfico en unas pocas áreas (general- 
mente de carácter urbano y rural urbanizadas) y existencia de altas tasas de masculinidad; también explica la inflexión de otras, como mudanza de signo en los saldos migratorio y, especialmente, demográfico, mejoría relativa del saldo vegetativo y de la tasa de envejecimiento y aumento de la tasa de actividad; finalmente, su contribución es vital para el mantenimiento de algunos servicios públicos, como la educación primaria, y de los equipamientos comerciales básicos. Sobre estos aspectos pasamos a centrar nuestra atención.
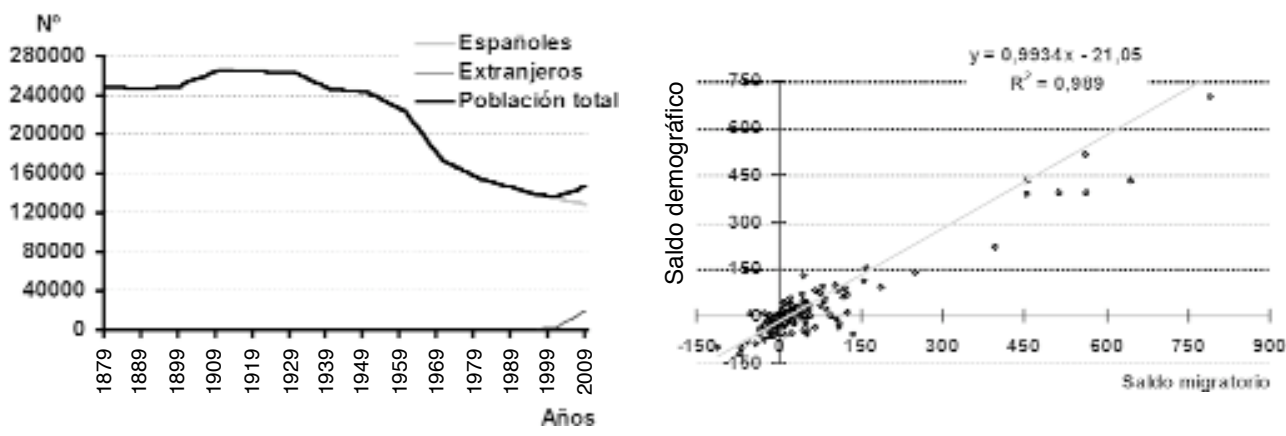

Figura 3. Evolución de la población (A) y relación entre los saldos migratorio y demográfico en los municipios turolenses entre 2001-2008 (excluidos Teruel y Alcañiz) (B). Elaboración propia.

La contribución al crecimiento de la población es el aspecto más destacable, si bien, por afectar sólo a los últimos ocho años (vid figura 3A), consideramos que es un lapso insuficiente para pensar en una ruptura definitiva con las pérdidas y más cuando la población española mantiene la trayectoria regresiva de siempre; en consecuencia, cualquier reducción del ritmo e intensidad en la llegada de extranjeros o, en la peor de las hipótesis, la inversión del proceso (como ha ocurrido en 2009, con un saldo migratorio de -559), puede dar al traste con la perspectiva de recuperación (de hecho, en el avance del padrón para 2010, a escala provincial, ya se constata una disminución de la población total). En términos espaciales, tomando el año 1991 como testigo, hasta 2008 sólo se ha producido incremento en el ámbito urbano, mientras en el rural intermedio y, sobre todo, en el desfavorecido, la población ha seguido disminuyendo (vid tabla 1), porque la inmigración no ha compensado un saldo vegetativo cada vez más negativo. 
Tabla 1. Saldos migratorio y vegetativo, según categoría de ruralidad. Elaboración propia.

\begin{tabular}{|l|l|c|c|c|c|c|}
\hline \multirow{2}{*}{$\begin{array}{l}\text { Valores } \\
\text { medios }\end{array}$} & \multirow{2}{*}{ Variable } & \multirow{2}{*}{ Urbano } & \multicolumn{4}{|c|}{ Rural } \\
\cline { 4 - 7 } & & Urbanizado & Dinámico & $\begin{array}{c}\text { Inter- } \\
\text { medio }\end{array}$ & $\begin{array}{c}\text { Desfavo- } \\
\text { recido }\end{array}$ \\
\hline \multirow{3}{*}{ 1991-1993 } & Población & 41.582 & 17.740 & 21.383 & 16.269 & 46.655 \\
& Saldo migratorio $\%$ & 2,4 & $-6,2$ & $-5,6$ & $-1,3$ & $-5,6$ \\
& Saldo natural $\%$ & 0,9 & 2,1 & $-1,8$ & $-6,0$ & $-9,4$ \\
\hline \multirow{3}{*}{$2006-2008$} & Población & 50.071 & 18.234 & 21.824 & 15.001 & 39.047 \\
& Saldo migratorio $\%$ & 20,2 & 19,9 & 18,8 & 12,0 & 6,2 \\
& Saldo natural $\%$ & 2,3 & $-1,3$ & $-2,6$ & $-7,9$ & $-9,8$ \\
\hline
\end{tabular}

La incidencia sobre la estructura demográfica también es interesante. Ha colaborado en el incremento de la tasa de masculinidad, por la importante llegada de hombres que viajan solos; los valores medios para el grupo de extranjeros han pasado de 161,4 en 2001 a 152,4 en 2008, en ambos casos muy altos, aunque con una ligera mejoría derivada del reagrupamiento familiar, pero, por colectivos, el máximo $(305,2)$ corresponde a los de origen africano y el mínimo $(84,6)$ a los americanos; los de la población española son de 101,7 y 102,6, respectivamente y los de la población total de 102,2 y 107,4. Pero, sobre todo, ha facilitado el control del deterioro del grupo humano, tanto por el lado de del envejecimiento (el 23,6 \% de la población total tiene 65 o más años en 2008 y entre la de nacionalidad española el 27,1\%), como en lo relacionado con la población de menos de 16 años (13,1\% para el total, 12,7 para los españoles y 16,9 para los extranjeros); el mayor envejecimiento corresponde a los municipios de menos de 500 habitantes, en su mayoría pertenecientes a las categorías de rural desfavorecido e intermedio, que tienen menos extranjeros empadronados y/o partían de una estructura demográfica inicial más deteriorada; la mayor presencia relativa de población de menos de 16 años se observa en los dos nodos urbanos, Teruel y Alcañiz (vid tabla 2).

La contribución a la mejoría del saldo natural, por el contrario, es menos perceptible, porque en la estructura del grupo inmigrante existe un déficit de población femenina en edad reproductora; con todo, aunque los comportamientos natalicios de ambos grupos son cada vez más parecidos (la diferencia ha disminuido de 2,4 veces en el 2000 a 1,5 en 2005, según IOE, 2008), a la población extranjera se debe cerca del $35 \%$ del esfuerzo natalicio total (lo que explicamos por su mayor tasa de fecundidad y menor envejecimiento), circunstancia que han servido para que la tasa de natalidad haya pasado del 6,2 \% en 2002 al 8,5 en 2008; la de mortalidad ha transitado de un 12,7 en 2003 a un 11,3\% en 2008. En consecuencia, el saldo sigue siendo negativo (salvo en la comarca del Bajo Aragón). En realidad, este factor sigue causando disminución de población y deterioro de la estructura por edades en casi un 
Tabla 2. Estructura demográfica, en 2008. Elaboración propia a partir de INE.

\begin{tabular}{|l|c|c|c|c|c|c|c|}
\hline \multirow{2}{*}{} & \multicolumn{3}{|c|}{ Población } & \multicolumn{2}{c|}{$>64$ años } & \multicolumn{2}{c|}{$<16$ años } \\
\cline { 2 - 7 } & Total & Extranj. & \% extran & Total & Extranj. & Total & Extranj. \\
\hline Según número de & & & & & & & \\
habitantes del municipio & & & & & & & \\
$100-499$ & 4.717 & 271 & 5,7 & 42,1 & 0,7 & 4,8 & 14,8 \\
$500-999$ & 23.741 & 2.529 & 10,6 & 34,2 & 0,9 & 9,4 & 20,4 \\
$1.000-2.499$ & 18.870 & 2.392 & 12,7 & 29,5 & 1,5 & 11,5 & 18,8 \\
$2.500-9.999$ & 17.615 & 2.295 & 13,0 & 25,2 & 0,9 & 12,8 & 18,1 \\
$\geq 10.000$ & 30.020 & 4.241 & 14,1 & 19,0 & 0,7 & 14,5 & 18,4 \\
\hline Según categoría territorial & 51.788 & 6.641 & 12,8 & 17,0 & 1,0 & 16,5 & 18,1 \\
Urbano & & & & & & & \\
Rural urbanizado & 51.788 & 6.641 & 12,8 & 17,0 & 1,0 & 16,5 & 18,1 \\
Rural dinámico & 18.744 & 2.480 & 13,2 & 18,5 & 0,8 & 14,8 & 17,7 \\
Rural intermedio & 22.416 & 3.510 & 15,7 & 21,3 & 0,6 & 13,7 & 18,4 \\
Rural desfavorecido & 15.091 & 1.990 & 13,2 & 28,2 & 0,9 & 11,9 & 19,2 \\
Total provincia & 38.712 & 3.748 & 9,7 & 34,4 & 1,4 & 9,2 & 19,6 \\
\hline
\end{tabular}

$70 \%$ del territorio provincial, lo que se visualiza con claridad en los gráficos de la figura 6; se ha producido un desplazamiento de las unidades territoriales desde el segundo y tercer cuadrantes hacia el cuarto, con dos excepciones, los municipios de menos de 100 habitantes, que siguen exhibiendo una ubicación indicadora de deterioro demográfico similar a la de los años noventa, y los de más de 10.000, o urbanos, que son los únicos que siguen estando en el primer cuadrante. Pero comarcas como Gúdar-Javalambre, Maestrazgo, Matarraña y Bajo Martín y todos los municipios de menos de 100 habitantes han seguido perdiendo población.

La influencia sobre la distribución de la población también es reseñable; dicha afirmación nos sugiere varias reflexiones. Su asentamiento es territorialmente heterogéneo, lo que explica que los impactos demográficos sean dispares; aunque el valor medio de participación es de un 12,5 \% sobre la población total, no son infrecuentes algunos por encima del 20, coincidiendo con pequeños municipios de menos de 250 habitantes. Sólo en 39 de los 236 municipios turolenses no reside ningún inmigrante extranjero, curiosamente, casi todos por debajo de ese umbral de habitantes. Este parece ser el límite que marca el umbral demográfico mínimo que induce mayor presencia de este tipo de población; pero esta conclusión sirve a escala municipal y no tanto a la de núcleo de población, puesto que hay casos, como Teruel y Calamocha, en los que la ubicación de extranjeros en núcleos diferentes a la cabecera municipal está reforzando la suburbanización. Por otra parte, ha ayudado a fortalecer la tendencia a la concentración de la población, porque es en los de mayor tamaño, nor- 
malmente más dinámicos en clave económica, donde se acumulan las mayores cifras absolutas (índice de Gini en 2008=0,26 para los inmigrantes de origen extranjero); desde este punto de vista, si bien colabora en el mantenimiento de la estructura actual de asentamientos, también es cierto que las tendencias de fondo ya preexistentes en el modelo de distribución de la población, según Frutos et alii (1994), no han cambiado; antes bien, algunas se han reforzado, nos referimos a la existencia de un número creciente de asentamientos de menos de 500 habitantes; en ellos sólo ha menguado la intensidad en el deterioro demográfico.
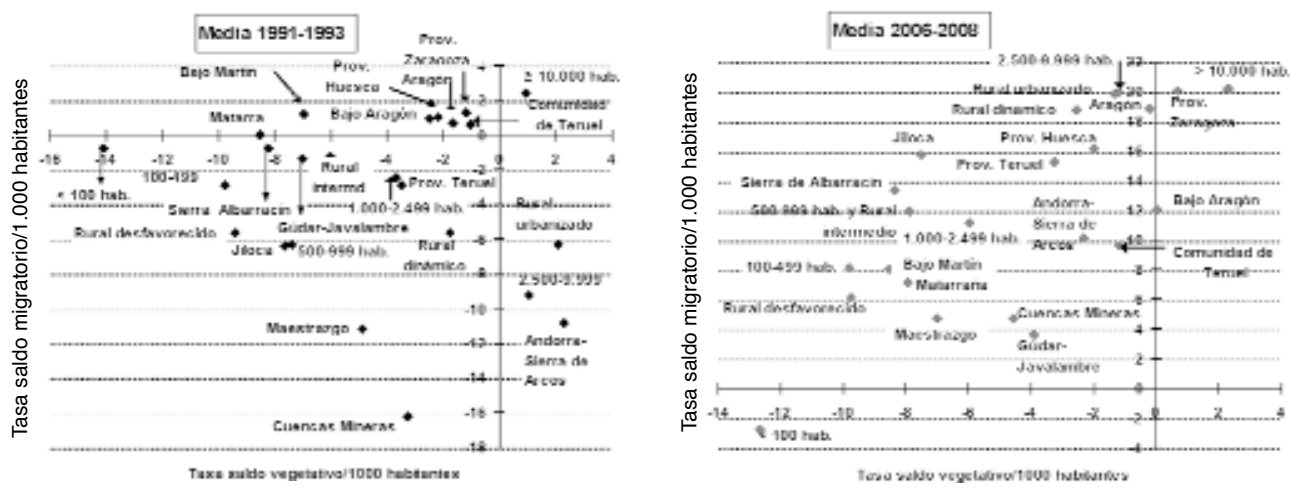

Figura 4. Diagramas de dispersión: saldos vegetativo/migratorio. Elaboración propia.

Finalmente, según categoría territorial, su contribución relativa a la mejora de la base de la pirámide demográfica es especialmente significativa en los ámbitos rural dinámico, intermedio y desfavorecido, en todos los casos mayor que en las áreas urbanas y rurales urbanizadas (vid tabla 2). Pero queremos destacar el rural desfavorecido, la parte demográficamente más sensible y deteriorada de ese territorio; en ella, la contribución al mantenimiento de los servicios básicos, tanto privados como públicos y de los equipamientos comerciales está siendo máxima, de lo que ha derivado un impacto positivo sobre la calidad de vida local; nos referimos a la reapertura de oferta comercial bajo la figura de multiservicios rurales ${ }^{3}$ (unos 25), en municipios que previamente la habían perdido.

\footnotetext{
${ }^{3}$ La red de Multiservicios Rurales gira en torno a la figura de "tienda-bar", que puede complementarse, además, con el servicio de restaurante, alojamiento, acceso a internet o información turística. Es un proyecto conjunto de la Cámara de Comercio e Industria de Teruel, la Diputación Provincial de Teruel y el Departamento de Industria, Comercio y Turismo del Gobierno de Aragón, cuyos objetivos son luchar contra el desabastecimiento en áreas rurales y ofrecer servicios fundamentales para la mejora de la calidad de la población. http://www.camarateruel.com/comercio_multiservicio.php
} 


\section{Reflexiones finales}

Nuestro trabajo entronca con un principio conceptual básico, la interdependencia entre población y actividad económica, que puede conformar desde un bucle que alimente el desarrollo, hasta configurar otro que cebe la crisis territorial. El crecimiento demográfico reciente tiene su base en la fuerte inmigración que ha experimentado la provincia, por la alta demanda de mano de obra derivada de un ciclo económico expansivo, que ha corregido el déficit en el mercado laboral local. La inmigración ha revitalizado el tejido demográfico, pero no sin incertidumbres, porque no se debe olvidar que buena parte de la demanda de mano de obra ha sido forzada por la evolución del sector de la construcción; la crisis actual que padece, unida a su alta capacidad de arrastre sobre el tejido económico provincial, hacen que seamos pesimistas sobre el mantenimiento a medio plazo de la dinámica inmigratoria. En realidad, a lo largo del último decenio, salvo la excepción de la agroindustria y el turismo rural, y aún éste con claroscuros, no ha habido génesis significativa de nuevo tejido productivo con visos de estabilidad, o, dicho de otro modo, cambio de modelo productivo; por ello, las tendencias demográficas de fondo se han mantenido constantes y la crisis pone en riesgo el signo positivo del balance migratorio.

Al ser un proceso excesivamente reciente, espontáneo y no exento de la oportunidad otorgada por la coyunturalidad, la principal incertidumbre que lo rodea es que el impulso demográfico que ha otorgado se agote en la parte más sensible del territorio turolense. Nos referimos a la formada por los municipios de menos de 500 habitantes, la más rural por su funcionalidad territorial, la que, salvo excepciones, tiene menor dinamismo extra-agrario y aquella que exhibe una estructura demográfica más deteriorada. Por supuesto, existen algunas excepciones ligadas a la agroindustria y al turismo rural; ambas actividades han dado lugar a un dinamismo que funciona como causa de atracción demográfica y factor de una mayor propensión al asentamiento de la población; cuando así ha sido, la modificación de la estructura demográfica tiene más visos de proyección futura, y lo mismo cabe indicar sobre los nodos urbanos y semiurbanos.

\section{Bibliografía}

Amuedo-Dorantes, C., y de la RICA, S. (2005). Immigrants. Responsiveness to Labor Market Conditions an its Implications on Regional Disparities: Evidence from Spain IZA Discussion Paper, 1557 (en línea). $<$ www.iza.org> (Consulta: 14.10.2010].

Bayona, J. y Gil, F. (2010). Migraciones de españoles y extranjeros en las áreas rura- les catalanas (1996-2006): ¿fin de la despoblación o nuevo modelo territorial? Boletín de la Asociación de Geógrafos Españoles, 53, 219-237.

Bel, G. y Gómez, J. (2001).La inmigración ¿problema o reto? Papeles de Geografía, 34, 51-67. 
Cánoves, G. y Blanco, A. (2009). Turismo, mercado de trabajo e inmigración en España: un análisis de la situación en las Comunidades de Murcia, Canarias, Valencia y Andalucía. Boletín de la Asociación de Geógrafos Españoles, 50, 259-280.

Carrasco, R. y Ortega, C. (2005). La inmigración en España: características y efectos sobre la situación laboral de los trabajadores nativos. Documento de trabajo no 0 (en línea). Madrid, Fundación Alternativas. $<$ http://www.almendron.com/politica/pdf /2006/spain/spain_3816.pdf> (Consulta: 6.9.2010)

Casado, R.M. (2009. La irrupción de España en el panorama de las migraciones internacionales. Por qué llegaron, por qué siguen llegando y por qué lo seguirán haciendo. ICE, 854, 5-22.

Cebrián, J.A. et alii (2010). La crisis económica internacional y sus repercusiones en España y en su población inmigrante. Estudios geográficos, Vol. 71, 268, 67-101

Cohen, A. (2009). España en la encrucijada migratoria (trans)-mediterránea. Una revisión sociogeográfica. Cahiers de civilisation espagnole contemporaine, $\mathrm{n}^{\mathrm{o}} 4$ (en línea).

<http://ccec.revues.org/index2430.html> (Consulta: 8/11/2010)

Colectivo IOE (2008). Inmigrantes, nuevos ciudadanos (en línea).

$<$ http://www.colectivoioe.org/uploads/b225a c1dc83b7bebdda55da3c4e7ef65a1cd9a72. pd> (Consulta: 9.11.2010).

Colectivo IOE (2010). Discursos de la población inmigrante en torno a su instalación en España. Exploración cualitativa. Centro de Investigaciones Sociológicas (en línea). <http://www.cis.es/cis/opencms/Archivos/Publicaciones/OyA/OyA64a.pdf > (Consulta: 22.11.2010).
Colectivo IOE y Fernández, M. (2010). Encuesta nacional de inmigrantes 2007: el mercado de trabajo y las redes sociales de los inmigrantes. Madrid, Ministerio de Trabajo e Inmigración.

Domínguez, R. (2003). Sin aumento de población no hay desarrollo económico. Enseñanzas de la historia económica regional de España. DOMINGUEZ, R. (ed) ¿Convergencia sin cohesión territorial? Teruel y otros desiertos demográficos. Teruel, Ed. Fundación Teruel Siglo XXI.

Frutos, L. M. et alii (1994). Cambios en el sistema de asentamientos rurales. La provincia de Teruel". Geographicalia, 31, 83-94.

García Sanz, B. (2006). Inmigración extranjera y ruralidad. Circunstancia: revista de ciencias sociales del Instituto Universitario de Investigación Ortega y Gasset, $\mathrm{n}^{\circ} 10$ (en línea]. <http://www.fog.es/circunstan$\mathrm{cia} /$ numero10/art19.pdf> (Consulta: 8.11.2010).

González, J. (2008). La aparición reciente del fenómenos inmigratorio extranjero en Galicia. Características e impronta espacial. Boletín de la A.G.E., 48, 247-275.

IAEST (en linea) <www.ine.es/inebase>

INE (en línea) <www.ine.es/inebase>

Martín, P. et alii. (2007). Capital social e inmigración: conceptualización operativa de la inserción sociolaboral de los inmigrantes. Studium, 13, 257-281.

Oficina Económica del Presidente Español (2006). Inmigración y economía española, 15 de noviembre de 2006 [en línea]. $<$ http://www.ucm.es/info/ecap2/mulas_c arlos/economia_laboral/Tema6bisMovilidadInmigracionEspanaOEP.pdf $>$ (Consulta: 28.9.2010)

Rodríguez, Mํ. A. (2010). La importancia de la inmigración en una región sin tradición: Castilla La Mancha y Ciudad Real (1996- 
2006). Boletín de la Asociación de Geógrafos Españoles, 53, 287-307.

Roquer, S. y Blay, J. (2008). Del éxodo rural a la inmigración extranjera: el papel de la extranjería en la recuperación de las zonas rurales españolas (1996-2006). Geo Crítica. Cuadernos Críticos de Geografía Humana, Vol. XII, núm. 270 (129) [en línea]. <http://www.ub.edu/geocrit/sn/sn270/sn-270-129.htm>

(Consulta: 20/12/2010)

Sáez, L. A. (2007). La economía de la Sierra de Albarracín. Zaragoza, Centro de Estudios sobre la Repoblación y Desarrollo de Áreas Rurales-Comunidad de Albarracín.

Sáez, L. A. et alii (2008). "Políticas inmigratorias frente a la despoblación: el caso de Aragón”. XV Encuentro de Economía Pública: políticas públicas y migración [en línea].

$<$ http://dialnet.unirioja.es/servlet/articulo?codi go $=3136560>$ (Consulta: 22.12.2010)

Serrano, J.M. (2006). Extranjeros En España: residentes e inmigrantes. Situación presente y perspectivas inmediatas. Anales de Historia Contemporánea, 22, 25-53. 